\title{
Concentrações de hormônio na carcaça de tilápias-do-nilo e maturação precoce após reversão sexual
}

\author{
Munir Francisco Zanardi ${ }^{1}$, Teresa Cristina Ribeiro Dias Koberstein ${ }^{2}$, Elisabeth Criscuolo \\ Urbinati $^{3}$, Michele Fagundes ${ }^{1}$, Marcio Alves dos Santos ${ }^{2}$, Maria Isabel Mataqueiro ${ }^{4}$ \\ ${ }^{1}$ Centro de Aquicultura - CAUNESP - UNESP, Jaboticabal, SP, 14884-900, Via de Acesso Paulo Castellane, s/n. \\ 2 CAUNESP - UNESP, Jaboticabal, SP. \\ ${ }^{3}$ CAUNESP e Faculdade de Ciências Agrárias e Veterinária - FCAV - UNESP, Jaboticabal, SP. \\ ${ }^{4}$ Faculdade de Ciências Agrárias e Veterinária - UNESP, Jaboticabal, SP.
}

RESUMO - Um total de 1.500 larvas de tilápia-do-nilo foi distribuído em 15 aquários de 20 L (100 larvas cada um) para comparação de dois métodos de masculinização: via oral, com dieta com hormônio (60 mg do $17 \alpha$-metiltestosterona.kg-1); e via banho de imersão (6 mg do $17 \alpha$-metiltestosterona. $\mathrm{L}^{-1}$ ), cada um com cinco repetições. As larvas e os juvenis foram amostrados no dia 1 (início do experimento) e aos 30 (final do período de alimentação com hormônio), 40, 45, 60 e 90 dias. Uma amostra de $0,5 \mathrm{~g}$ de peixe foi coletada em cada repetição para análise da testosterona corporal. Os peixes alimentados com a dieta com hormônio receberam ração experimental por 30 dias e ração comercial até o final do experimento, e banho de imersão receberam ração comercial e foram submetidos a banhos de imersão ( $6 \mathrm{mg}$ da $17 \alpha$-metiltestosterona. $L^{-1}$ ), de 36 horas, nos dias 6 e 10 após início do experimento. Nos peixes que receberam a ração sem hormônio (controle), os valores de testosterona corporal se mantiveram praticamente estáveis ao longo do experimento, aumentando moderadamente a partir de 60 dias. As concentrações de testosterona corporal nos peixes que receberam a dieta com hormônio ou o banho de imersão foram mais altas aos 30 dias. Nos peixes submetidos ao banho de imersão, os valores reduziram aos 40 dias e aumentaram novamente até os 60 dias de observação, enquanto naqueles submetidos à dieta com hormônio, as concentrações de testosterona aumentaram gradativamente até 60 dias. A utilização de $17 \alpha$-metiltestosterona por via oral ou banho de imersão das larvas estimula a maturação sexual dos peixes a partir dos 45 dias, especialmente naqueles alimentados com ração contendo hormônio. As concentrações desse hormônio na carcaça são inferiores ao preconizado pelo Codex Alimentarius do Brasil como seguras para consumo humano.

Palavras-chave: consumidor, dano à saúde, masculinização, metiltestosterona

\section{Hormone concentration in carcass of Nile tilapia submitted to early maturation after sexual reversion}

\begin{abstract}
A total of 1,500 larvae of Nile tilapia was distributed in 15 20-L aquaria (100 larvae in each one) to compare two methods of masculinization: via oral application, using a diet with hormone (60 mg $17 \alpha$-methyltestosterone. $\mathrm{kg}^{-1}$ ); and through immersion bath (6 mg $17 \alpha$-metyltestosterone. $\mathrm{L}^{-1}$ ), each one with five replicates. Larvae and juvenile were sampled on day 1 (beginning of the experiment) and on days 30 (end of hormone feeding period), 40, 45, 60 and 90. One sample with $0.5 \mathrm{~g}$ of fish was collected from each replication for analysis of body testosterone. Fish fed diet with hormone were given experimental ration for 30 days and commercial ration until the end of the experiment, and fish in immersion bath received commercial ration and they were submitted to immersion bath (6 mg $17 \alpha$-metyltestosterone. $\mathrm{L}^{-1}$ ) for 36 hours on days 6 and 10 after the beginning of the experiment. For fish given ration without hormone (control), values of body testosterone were almost totally steady over the experiment, moderately increasing from day 60. Concentrations of body testosterone in fish fed diet with hormone or immersion bath were the highest on day 30. For fish submitted to immersion bath, the values were reduced on day 40 and they increased again until 60 days of observation, while for those submitted to diet with hormone, concentrations of testosterone gradually increased until 60 days. The use of $17 \alpha$-methyltestosterone through oral administration or immersion bath of larvae promotes sexual maturation of fish from day 45, especially on those fed diet with hormone. Concentrations of hormone in the carcass are lower than the recommended by Codex Alimentarius from Brazil as safe for human consumption.
\end{abstract}

Key Words: consumer, damage to health, masculinization, metyltestosterone 


\section{Introdução}

A tilápia-do-nilo (Oreochromis niloticus) tem grande precocidade reprodutiva, o que representa um problema na piscicultura (Neumann et al., 2009). A precocidade reprodutiva causa superpopulação, que, para ser controlada, exige aplicação de alguns métodos, como a masculinização por meio de hormônios esteroides masculinizantes (Popma \& Green, 1990), tanto na ração como em banhos de imersão (Gale et al., 1999), para produção de população monossexual.

A adição do hormônio 17- $\alpha$-metiltestosterona na ração é o método mais utilizado na reversão sexual de tilápias e pode produzir 98\% de machos (Mainardes-Pinto et al., 2000) utilizando-se ração suplementada com metiltestosterona (60 mg. $\mathrm{kg}^{-1}$ ) por um período de 30 dias a partir do início da alimentação exógena das larvas. Banhos de imersão de larvas em solução contendo metiltestosterona para masculinização dos peixes, de acordo com outros autores (Bombardelli et al., 2007; Dias-Koberstein et al., 2007), resulta em populações com até $83 \%$ de machos.

Segundo Collins et al. (1989), anabolizantes como a testosterona e trembolona muitas vezes usados na produção animal, não são permitidos na Europa, fazendo com que todo produto importado e que apresente resíduos de hormônio seja embargado. Já nos Estados Unidos, é permitido o uso de testosterona e acetato de trembolona em produtos de origem animal.

No Brasil, foram proibidos a importação, produção e comercialização e o uso de substâncias naturais ou artificiais para crescimento e/ou engorda de animais de abate, com permissão apenas para fins terapêuticos e processos reprodutivos (Brasil, 1991). Em 1995, foi determinado pelo Codex Alimentarius do Brasil que a testosterona não apresentava problemas para a saúde humana, desde que em doses inferiores a 2 mg. .g $^{-1}$ (Palermo-Neto, 1998).

Com a alta produção de tilápia, é importante que o consumidor se assegure de que o produto a ser consumido esteja dentro dos padrões de segurança alimentar, sem riscos de efeitos residuais de hormônios esteroides. O objetivo neste trabalho foi determinar os efeitos do $17-\alpha-$ metiltestosterona por dois métodos de masculinização (alimentação com ração contendo o hormônio e banhos de imersão em solução enriquecida) sobre a maturação sexual e a presença de resíduos do andrógeno na carcaça de larvas e juvenis de tilápia-do-nilo.

\section{Material e Métodos}

Ovos de três reprodutores de tilápia-do-nilo, retirados da boca dos animais, foram misturados e incubados.
Após a absorção do saco vitelino, 1.500 larvas com comprimento e peso médio de $8,75 \mathrm{~mm} \pm 0,071$ e 0,0093 g \pm 0,00052, respectivamente, foram distribuídas em 15 aquários plásticos de $20 \mathrm{~L}$, com 100 indivíduos por aquário. Os foram instalados dentro de caixas plásticas de $175 \mathrm{~L}$, em banhomaria, a $30^{\circ} \mathrm{C} \pm 2^{\circ} \mathrm{C}$, com aeração contínua nos aquários.

Avaliou-se a masculinização com $17-\alpha$-metiltestosterona via ração e por meio de banho de imersão, em comparação a uma ração sem hormônio. Os tratamentos foram aplicados aleatoriamente nos 15 aquários, com cinco repetições por tratamento. À ração comercial fornecida aos grupos, adicionou-se $17 \alpha$-metiltestosterona (60 mg/kg de ração), que foi fornecida aos peixes durante 30 dias, seis vezes ao dia, até aparente saciedade. Os banhos de imersão foram realizados em aquários de vidro de 2,5 L, por 36 horas, em água contendo $6 \mathrm{mg} . \mathrm{L}^{-1}$. Os banhos aconteceram no $60 \mathrm{o} \mathrm{e}$ $10^{\mathrm{o}}$ dias do período de 30 dias. Após os banhos, as larvas que estavam nos vidros voltaram para os aquários de $20 \mathrm{~L}$. Ao final dos 30 dias, os juvenis foram transferidos para caixas de fibra e silicone, com capacidade de $200 \mathrm{~L}$, onde permaneceram por mais 60 dias, até o final do experimento. Nesse período, passaram a ser alimentados quatro vezes ao dia com ração comercial.

No início do experimento (dia 1) e aos 30 (final do período de oferecimento da ração com hormônio), 40, 45, 60 e 90 dias do período experimental, coletaram-se amostras de $0,5 \mathrm{~g}$ de larvas e juvenis, que foram congeladas em gelo seco e mantidas a $-20^{\circ} \mathrm{C}$ até quantificação de testosterona tecidual, de acordo com metodologia descrita por Jesus et al. (1991), modificada por Brinn (2003) e adaptada para extração do hormônio em larvas e juvenis de tilápia-do-nilo.

As amostras foram homogeneizadas em solução tampão salina com gelatina, misturadas com éter etílico em agitador e centrifugadas por cinco minutos para separação de sobrenadante. Os tubos contendo as amostras foram, então, colocados em gelo seco e acetona para melhor separação dos sobrenadantes, que foram separados em outros tubos. O procedimento foi repetido duas vezes e os tubos contendo os sobrenadantes foram colocados em banho-maria até evaporação completa do éter. Depois de completar a evaporação, adicionou-se a solução tampão aos tubos, que foram levados a um sonicador, para que o material aderido às paredes se desprendesse, e a amostra foi agitada novamente para dissolução completa.

A leitura das amostras foi feita por ELISA com o kit testosterona EIA (Interkit) e a unidade foi expressa em ngT.g ${ }^{-1}$ de carcaça.

Para determinar as variações intraensaio e interensaio e para o teste de recuperação do hormônio $(n=6)$, foram utilizadas soluções de testosterona contendo 3, 6, 9 e 
12 ng.mL ${ }^{-1}$. As variações intraensaio foram 28,54 e 86,67\%, e as variações interensaio foram 20,92 e 21,27\% para valores altos e baixos de testosterona, respectivamente, enquanto a recuperação do hormônio foi de $69,9 \%$.

A proporção de sexos foi determinada por análise microscópica das gônadas, utilizando-se a técnica do acetato-carmim.

O delineamento experimental utilizado foi inteiramente ao acaso com três tratamentos e cinco repetições, pelo programa estatístico SAS (Statistic Analysis System), versão 9.1. Os dados coletados para as variáveis foram submetidos à análise de variância (ANOVA) a 5\% de probabilidade e, em caso de diferenças significativas, foi aplicado o teste Tukey ( $\alpha=5 \%)$.

\section{Resultados e Discussão}

Os parâmetros da água monitorados foram $\mathrm{pH}$ (7,31 $\pm 0,03)$, condutividade $\left(297,9 \pm 3,39 \mathrm{mg} \cdot \mathrm{L}^{-1}\right)$, oxigênio dissolvido (5,41 $\left.\pm 0,03 \mathrm{mg} . \mathrm{L}^{-1}\right)$, alcalinidade $\left(195 \pm 2 \mathrm{mg} . \mathrm{L}^{-1}\right)$, amônia (80,63 $\left.\pm 15,58 \mathrm{mg} . \mathrm{L}^{-1}\right)$, sólidos disponíveis totais $\left(0,27 \pm 0,01 \mathrm{~g} . \mathrm{L}^{-1}\right)$, salinidade $\left(0,157 \pm 0,003 \mathrm{mg} . \mathrm{L}^{-1}\right) \mathrm{e}$ temperatura $\left(30,14 \pm 0,27^{\circ} \mathrm{C}\right)$, que estiveram de acordo com o recomendado para tilapicultura por Popma \& Phelps (1998).

A proporção de machos obtida com a ração sem hormônio foi de $68,00 \% \pm 13,03$, com a ração contendo hormônio 94,00\% \pm 8,94 e com o banho de imersão 86,00\% $\pm 5,47$ após 90 dias do início do experimento. No primeiro dia do experimento, três pools de 150 larvas, correspondendo à concentração inicial de testosterona corporal em cada grupo, apresentaram $0,51 \mathrm{ngT}^{-1}{ }^{-1}$. Ao final de 30 dias, nos quais as larvas receberam ração sem hormônio, ração com hormônio ou banho de imersão, as concentrações de testosterona tecidual aumentaram $(0,63 \pm 0,32 ; 1,56 \pm 1,27$ e 2,67 \pm 0,44 ngT. $\mathrm{g}^{-1}$, respectivamente). Aos 40 dias, os valores registrados para ração sem hormônio, ração com hormônio e banho de imersão, respectivamente, foram $0,61 \pm 0,13 ; 1,78 \pm 0,42$ e $0,79 \pm 0,24$ ngT.g ${ }^{-1}$; aos 45 dias, $0,69 \pm 0,45 ; 2,32 \pm 0,73$ e 1,68 $\pm 0,89$ ngT.g ${ }^{-1}$; aos 60 dias, $1,02 \pm 0,70 ; 2,66 \pm 1,96$ e $2,31 \pm 0,95 \mathrm{ngT} \mathrm{g}^{-1}$; e aos 90 dias $1,21 \pm 0,99 ; 2,81 \pm 0,38$ e $2,31 \pm 0,63$ ngT.g ${ }^{-1}$.

No grupo alimentado com ração sem hormônio, as concentrações de testosterona na carcaça aumentaram moderadamente ao longo do período experimental, especialmente após os 60 dias $(0,36 ; 0,63 ; 0,61 ; 0,69 ; 1,02$; 1,21 ngT.g ${ }^{-1}$ ). Já no grupo que recebeu a ração com hormônio, houve aumento de quase três vezes em relação ao controle 30 dias após a alimentação com ração suplementada com metiltestosterona $\left(0,67 ; 1,56\right.$ ngT.g $\left.{ }^{-1}\right)$. Esses valores aumentaram gradativamente até os 90 dias $(1,78 ; 2,32 ; 2,66$; 2,81 ngT.g ${ }^{-1}$ ). No banho de imersão, as concentrações mais altas de testosterona nos tecidos ocorreram no $30^{\circ}$ dia (2,67 ngT.g $\left.{ }^{-1}\right)$, que reduziram no $40^{0}$ dia $\left(0,79\right.$ ngT.g $\left.{ }^{-1}\right)$ e voltaram a aumentar a partir de 45 dias $(1,68 ; 2,31$; 2,31 ngT.g ${ }^{-1}$ ) (Figura 1).

Os grupos que receberam a ração com hormônio e a controle tiveram mesmo perfil de testosterona corporal, embora as concentrações tenham sido muito mais altas nas larvas tratadas com metiltestosterona. Considerando que 0 hormônio exógeno foi retirado da alimentação aos 30 dias, o hormônio detectado, em concentrações crescentes, embora em níveis moderados, parece ser de origem endógena. Nas larvas submetidas aos banhos de imersão com metiltestosterona, a concentração de testosterona aos 30 dias foi quase o dobro da observada nas larvas tratadas com ração com hormônio, embora o último banho tenha ocorrido 20 dias antes. Não se trata, portanto, de produção endógena, visto que, aos 40 dias, essa concentração caiu

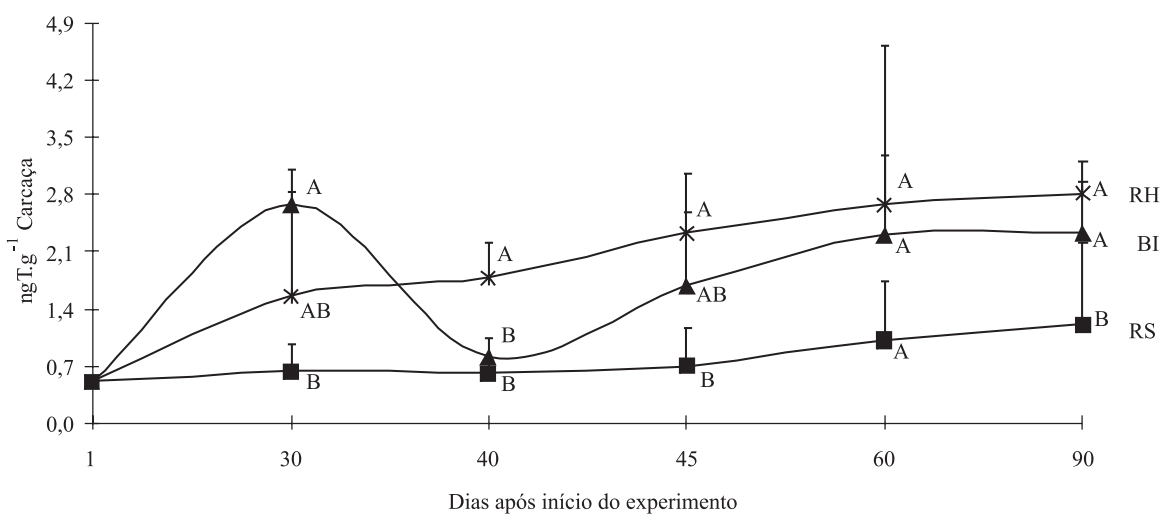

Figura 1 - Concentrações de testosterona nos tecidos de tilápias-do-nilo submetidas a maturação precoce após banhos de imersão. 
a 1/3 do registrado aos 30 dias e em seguida teve mesmo comportamento de aumento verificado nos outros grupos. Como não houve amostragem para determinação de testosterona corporal logo após os banhos de imersão, não é possível discutir os valores registrados aos 30 dias, mesmo porque não há na literatura estudos sobre o mecanismo de absorção, a farmacocinética e a biodisponibilidade de metiltestosterona em imersão em peixes. O fornecimento de metiltestosterona a trutas por via oral comprovou que o andrógeno tem disponibilidade de $70 \%$ e meia-vida de 4,7 horas, enquanto a meia-vida de eliminação de metiltestosterona administrada intra-arterialmente ( $20 \mathrm{mg} / \mathrm{kg}$ ) foi de 58 horas (Vick \& Hayton, 2001). Isso reforça a dificuldade de explicar o valor de testosterona encontrado aos 30 dias nas larvas expostas a metiltestosterona por banho de imersão.

A diferença nas concentrações de testosterona pode ser atribuída ao número de machos revertidos em cada grupo experimental. No grupo alimentado com ração contendo hormônio, em que foram observados 94\% de machos, as concentrações foram mais altas; no grupo submetido ao banho de imersão a reversão foi de $86 \%$, as concentrações foram intermediárias e no grupo controle em que a reversão foi de apenas $68 \%$, ocorreram os valores mais baixos de testosterona. Em estudo sobre identificação de esteroides sexuais em dietas comerciais para salmões, Sower \& Iwamoto (1985) observaram que o uso de andrógenos sintéticos ou naturais na ração pode influenciar o desenvolvimento gonadal dos peixes, acelerando a espermatogênese e aumentando a precocidade sexual em machos.

Nesta pesquisa, o aumento dos níveis hormonais na carcaça dos peixes sugere maturação mais precoce da espécie. Numa criação natural, em viveiros de tilápias, o início de reprodução ocorre 3 a 4 meses após a estocagem dos alevinos, em ambos os sexos. Entretanto, havia 100\% de machos (Zimmermann, 1999).

Nas condições experimentais deste estudo, considerando a ocorrência de maturação sexual e produção endógena de andrógenos pelos juvenis de tilápia, não foram detectados hormônios exógenos residuais. Entretanto, o hormônio exógeno desapareceu da carcaça dos alevinos de tilápia, uma vez que, em estudos prévios, Gouldie et al. (1986) alimentaram, por 21 dias, larvas de Oreochromis aureus com rações contendo $17-\alpha$-metiltestosterona marcada com trício e $\mathrm{C}^{14}$ e investigaram a distribuição da radioatividade nos músculos e nas vísceras e a taxa de eliminação da radiação. Radioatividade foi encontrada na carcaça uma hora após a alimentação inicial e atingiu os valores mais altos em seis horas. Mais de $90 \%$ da radioatividade estava presente nas vísceras durante os 21 dias de alimentação e foi eliminada exponencialmente, diminuindo $90 \%$ em 24 horas depois de finalizada a alimentação com ração marcada, tanto nas vísceras como nos músculos. Em outro estudo, studo previnos, levando em consideraRothbard et al. (1990) mediram, nos músculos de larvas de tilápias (Oreochromis aureus $\times$ O. aureus) alimentadas por 11 semanas com ração contendo 17- $\alpha$-etiniltestosterona (60 metiltestosterona. $\mathrm{kg}^{-1}$ ), a eliminação do andrógeno em razão do tempo. Os autores só encontraram quantidades detectáveis do hormônio no primeiro dia após a finalização da alimentação com o andrógeno. As concentrações em amostras retiradas no $3^{0}$, $5^{\circ}$ e $7 \stackrel{0}{0}$ dias não diferiram daquelas observadas nos peixes não-tratados e estavam abaixo de 50 ng.g ${ }^{-1}$. Portanto, a masculinização de tilápias com administração de metiltestosterona via oral ou por banho de imersão das larvas não traz risco para a saúde dos consumidores, pois, além da eliminação rápida da carcaça, as concentrações detectadas são inferiores aos valores preconizados como seguros pelo Codex Alimentarius do Brasil em 1995, de 2 mg. $\mathrm{kg}^{-1}$ (Palermo-Neto, 1998).

\section{Conclusões}

As concentrações de testosterona tecidual aumentam com a ingestão e/ou absorção do hormônio em banho de imersão. Quando o hormônio é aplicado via banho de imersão, suas concentrações máximas reduzem a valores próximos do observado com alimentação controle, sem hormônio, o que indica capacidade dos peixes de eliminar a testosterona em até dez dias. No caso da alimentação com o hormônio, não ocorre essa redução.

\section{Agradecimentos}

Aos Laboratórios de Fisiologia de Peixes e de Farmacologia do Departamento de Morfologia e Fisiologia Animal - Faculdade de Ciências Agrárias e Veterinária, campus de Jaboticabal (UNESP) e Laboratório de Fisiologia Animal (LAFA), do Departamento de Ciências Básicas da Universidade de São Paulo - Faculdade de Zootecnia e Engenharia de Alimentos, campus de Pirassununga.

À Coordenação de Aperfeiçoamento de Pessoas de Nível Superior-CAPES.

\section{Referências}

BOMBARDELLI, R.A.; SANCHES, E.A.; PINTO, D.F.H. et al. Idade de maior sensibilidade de tilápia-do-nilo aos tratamentos de masculinização por banhos de imersão. Revista Brasileira de Zootecnia, v.36, n.1, p.1-6, 2007. 
BRASIL. Ministério da Agricultura. Portaria $n^{\circ} 51$ de 24 de maio de 1991. Diário Oficial da União, Brasília, 27 de maio de 1991. Seção I. p.9989.

BRINN, R.P. Cortisol exógeno em matrinxã (Brycon cephalus, Characidae, Günther, 1869): variáveis fisiológicas na maturação final e crescimento inicial da prole. 2003. 86f. Tese (Doutorado em Aqüicultura) - Centro de Aqüicultura da Universidade Estadual Paulista - CAUNESP, Jaboticabal.

COLLINS, S.S.; BELK, K.E.; CROSS, H.R. The EEC ban against growth-promoting hormones. Nutrition Reviews, v.47, n.8, p.238-246, 1989.

DIAS-KOBERSTEIN, T.C.R.; GABRIEL NETO, A.; STÉFANI, M.V. et al. Reversão sexual de larvas de tilápia-do-nilo (Oreochromis niloticus) por meio de banhos de imersão em diferentes dosagens hormonais. Revista Acadêmica, v.5, n.4, p.391-395, 2007.

CARMO, J.L.; FERREIRA, D.A.; DA SILVA R.E. et al. Crescimento de três linhagens de tilápia sob cultivo semi-intensivo em viveiros. Caatinga (Mossoró, Brasil), v.21, n.2, p.20-26, 2008.

GOUDIE, C.A.; SHELTON, W.L. PARKER, N.C. Tissue distribution and elimination of radiolabelled methyltestosterone fed to sexually undifferentiated bleu tilapia. Aquaculture, v.58, n.3/4, p.215-226, 1986.

JESUS, E.G.; HIRANO, T.; INUI, Y. Changes in cortisol and thyroid hormone concentrations during early development and metamorphosis in the Japanese flounder, Paralichthys olivaceus. General and Comparative Endocrinology, v.82, n.1 p.369-376, 1991.

MAINARDES-PINTO, C.S.R.; FENERICH-VERANI, N.; DE CAMPOS, B.E.S. et al. Masculinização da tilápia do nilo, Oreochromis niloticus, utilizando diferentes rações e diferentes doses de 17 a-metiltestosterona. Revista Brasileira de Zootecnia, v.29, n.3, p.654-659, 2000.

NEUMANN, E.; DIAS KOBERSTEIN, T.C.R.; DE SOUZA BRAGA, F.M. Desempenho de três linhagens de tilápia submetidas ao tratamento com 17- $\alpha$-metiltestosterona em condições ambientais não controladas. Revista Brasileira de Zootecnia, v.38, n.6, p.973-979, 2009.

PALERMO NETO, J. Anabolizante e pecuária de corte. Revista Educação Continuada - CRMV, v.1, n.1, p.10-15, 1998.

POPMA, T.J.; PHELPS, R.P. Status report to commercial tilapia producers on monosex fingerling productions techniques. In: AQUICULTURA BRASIL'98, 1998, Recife. Anais... Recife: SIMBRAQ, 1998. p.127-145.

POPMA, T.J.; GREEN, B.W. Reversão sexual de tilápias em tanques de terra. In: _-_-_ Manual de produção em aquacultura. Auburn: University Auburn, 1990. 52p.

ROTHBARD, S.; ZOHAR, Y.; ZMORA, N. et al. Clearance of 17-á-ethynyltestosterone from muscle of sex-inversed tilapia hybrids treated for growth enhancement with two doses of the androgen. Aquaculture, v.89, n.3/4, p.365-376, 1990 .

SOWER, S.A.; IWAMOTO, R.N. The identification of sex steroid, testosterone in various commercial salmon diets. Aquaculture, v.49, n.1, p.11-17, 1985.

VICK, A.M.; HAYTON, W.L. Methyl testosterone pharmacokinetics and oral bioavailability in rainbow trout (Oncorhynchus mykiss). Aquatic Toxicology, v.52, p.177-188, 2001.

ZIMMERMANN, S. Incubação artificial - Técnica permite a produção de tilápias-do-nilo geneticamente superiores. Panorama da Aquicultura, v.9, n.54, p.15-21, 1999. 